\title{
Caracterización molecular de aislamientos de Shigella sonnei recuperados en el programa de vigilancia por el laboratorio de la enfermedad diarreica aguda en Colombia
}

\author{
Nórida Vélez, Paula Lucía Díaz, Catering Rodríguez, Adriana Bautista, \\ Lucy Angeline Montaño, María Elena Realpe \\ Grupo de Microbiología, Instituto Nacional de Salud, Bogotá, D.C., Colombia
}

Introducción. En Colombia, Shigella sonnei es uno de los serotipos más frecuentemente aislados $(53,4 \%)$ de muestras clínicas humanas asociadas a la enfermedad diarreica aguda. La identificación de patrones de restricción del ADN mediante electroforesis en gel de campo pulsado constituye la base de la vigilancia molecular de S. sonnei.

Objetivo. Establecer la base de la vigilancia molecular de $S$. sonneien Colombia mediante electroforesis en gel de campo pulsado.

Materiales y métodos. Se estudiaron 102 de los 2.048 aislamientos de S. sonnei remitidos por la Red Nacional de Laboratorios entre 1997 y marzo del 2013; la selección se hizo de acuerdo con el patrón de resistencia antimicrobiana, el origen de la muestra y la relación con brotes. Se determinó el patrón genético mediante electroforesis en gel de campo pulsado con las enzimas de restricción Xbal y BInl, según el protocolo de la red PulseNet International. El análisis de los patrones electroforéticos se hizo con el programa GelCompar II, versión 4.0.

Resultados. Se obtuvieron 42 patrones electroforéticos con una similitud de 70 a $100 \%$. El patrón más frecuente fue COIN08J16X01.0017 (17,6 \%), seguido por los patrones COIN04J16X01.0004 (9,8\%) y COIN02J16X01.0002 (5,8\%), y el 66,8 \% restante se asoció con otros patrones electroforéticos. El análisis de brotes demostró la relación genética de cada brote con $100 \%$ de similitud en la identificación; el patrón más frecuente en los brotes fue el COIN08J16X01.0017 (17,1\%).

Conclusión. Se estableció la base de datos genotípicos de aislamientos de S. sonnei a nivel nacional mediante electroforesis en gel de campo pulsado; se incluyeron los 42 patrones únicos identificados en este estudio.

Palabras clave: Shigella sonnei, electroforesis en gel de campo pulsado, vigilancia epidemiológica, Colombia.

doi: http://dx.doi.org/10.7705/biomedica.v35i3.2622

\section{Molecular characterization of Shigella sonnei isolates recovered by the Laboratory Surveillance Program for Acute Diarrheal Disease in Colombia}

Introduction: In Colombia, Shigella sonnei is one of the most frequently isolated serotypes (53.4\%) in human clinical samples associated with diarrheal acute disease. The identification of DNA restriction patterns by pulsed field gel electrophoresis is the basis for the molecular surveillance of $S$. sonnei.

Objective: To establish the basis for the molecular surveillance of $S$. sonnei in Colombia using pulsedfield gel electrophoresis.

Materials and methods: We studied 102 of 2,048 S. sonnei isolates referred by the National Laboratory Network between 1997 and March, 2013; the selection was made according to the antimicrobial multiresistance profile, the source of samples, and the relation to outbreaks. The genetic profile was determined by pulsed field gel electrophoresis using the restriction enzymes Xbal and BInl in accordance with the PulseNet International protocol. The electrophoretic patterns were analyzed with the GelCompare II, version 4.0 software.

Results: We obtained 42 electrophoretic patterns with a $70 \%$ to $100 \%$ similarity. The most frequent pattern was COIN08J16X01.0017 with $17.6 \%$, followed by patterns COIN04J16X01.0004 with

\footnotetext{
Contribución de los autores:

Nórida Vélez y Paula Lucía Díaz: formulación del estudio, aplicación de la técnica molecular de PFGE y escritura del manuscrito Paula Lucía Díaz: estandarización de la técnica molecular de PFGE

Catering Rodríguez: desarrollo de la técnica de sensibilidad antimicrobiana

Adriana Bautista, Lucy Angeline Montaño y María Elena Realpe: identificación bioquímica y serotipificación

María Elena Realpe: coordinación de las actividades del programa de vigilancia por el laboratorio de la enfermedad diarreica aguda del Grupo de Microbiología

Todos los autores participaron en el análisis de resultados y la revisión del manuscrito.
} 
$9.8 \%$, and COIN02J16X01.0002 with 5.8\%, while the remaining $66.8 \%$ was associated with other electrophoretic patterns. The analysis of 10 outbreaks demonstrated their genetic relation with a $100 \%$ of similarity; the most frequent pattern in outbreaks was COIN08J16X01.0017 with $17.1 \%$.

Conclusion: The genotypic database for Shigella sonnei isolates was established using pulsed field gel electrophoresis including the 42 unique patterns identified in this study.

Key words: Shigella sonnei, electrophoresis, gel, pulsed-field, epidemiological surveillance, Colombia. doi: http://dx.doi.org/10.7705/biomedica.v35i3.2622

Shigella spp. es uno de los principales agentes patógenos causantes de enfermedad diarreica aguda. La shigelosis afecta principalmente a niños menores de cinco años, ancianos y personas inmunocomprometidas, y es uno de los principales problemas de salud a nivel mundial, especialmente en países en desarrollo con condiciones deficientes de higiene y con mala calidad en el suministro de agua (1-3).

Anualmente ocurren aproximadamente 165 millones de casos de shigelosis a nivel mundial, de los cuales el $99 \%$ se reporta en países en desarrollo y $0,91 \%$ en países industrializados; se estima que cada año 1,1 millones de personas mueren por infecciones asociadas a Shigella spp. Cerca de 580.000 casos de shigelosis se notifican entre los viajeros procedentes de países industrializados y el $60 \%$ de las muertes ocurre en el grupo de menores de cinco años (4). La resistencia antimicrobiana de Shigella spp. ha llevado a introducir cambios en su manejo terapéutico en la mayor parte del mundo, lo cual ha reducido los tratamientos de elección (5).

El tratamiento antimicrobiano para las infecciones asociadas con Shigella spp. incluye la ampicilina, el trimetoprim-sulfametoxazol y el ácido nalidíxico, pero debido al aumento a nivel mundial de la resistencia de Shigella spp. a la mayoría de estos antibióticos (6), se recomienda la ciprofloxacina como el medicamento de elección para pacientes con shigelosis de cualquier grupo de edad $(7,8)$.

Otras alternativas incluyen la ceftriaxona y la azitromicina, antimicrobianos efectivos para los aislamientos multirresistentes. Sin embargo, la resistencia de los aislamientos de Shigella spp. a

\footnotetext{
Correspondencia:

Paula Lucía Díaz, Grupo de Microbiología, Instituto Nacional de Salud, Avenida calle $26 N^{\circ}$ 51-20, Bogotá, D.C., Colombia Teléfono: (571) 220 7700, extensiones 1420 y 1421; fax: (571) 2207700 , extensión 1421

pdiaz@ins.gov.co

Recibido: 05/11/14; aceptado: 27/04/15
}

las fluoroquinolonas en India y en otros países asiáticos, limita la efectividad de estos antimicrobianos en el tratamiento de la shigelosis (9-12).

Shigella sonnei es responsable de la disentería y se considera un problema de salud pública emergente a nivel global (13), y en algunos países como Malasia e India, se ha observado un incremento en la dominancia de S. sonnei como agente etiológico de la shigelosis $(14,15)$.

Según los reportes de otros países de Latinoamérica, como Argentina, el cambio en la frecuencia del serotipo $S$. sonnei se comenzó a evidenciar a partir del 2003 (16). La importancia de Shigella spp. en Colombia se refleja en el hecho de que hasta julio de 2014 los laboratorios de salud pública del país habían notificado 4.415 aislamientos al sistema de vigilancia de enfermedades trasmitidas por alimentos y al de vigilancia de la enfermedad diarreica aguda que coordina el Grupo de Microbiología del Instituto Nacional de Salud. Desde 1997 y hasta julio del 2014, los serotipos más frecuentes reportados fueron S. sonnei $(54,7 \%)$, Shigella flexneri (42\%), Shigella boydii (2,2\%), y Shigella dysenteriae $(0,2 \%)$; aquellos cuyos serotipos no pudieron identificarse representaron el 0,8\% (17).

La sensibilidad antimicrobiana encontrada en 2.418 aislamientos de S. sonnei entre 1997 y julio del 2014, se asoció a trimetroprim-sulfametoxazol (88\%), tetraciclina (88,3\%), ampicilina $(47,7 \%)$, cloranfenicol (23,7\%), amoxicilina y ácido clavulánico $(6,4 \%)$ (datos sin publicar del Grupo de Microbiología-Red Nacional de Laboratorios, Instituto Nacional de Salud).

La técnica de electroforesis en gel de campo pulsado (PFGE) es un método de tipificación molecular con protocolos estandarizados que permiten comparar el ADN bacteriano entre aislamientos y hacer el seguimiento de patrones moleculares a nivel regional o internacional (18).

A partir del 2004, la vigilancia molecular de Salmonella spp. mediante la técnica de PFGE se incorporó a la Red PulseNet de América Latina y 
el Caribe; en el 2012 el Grupo de Microbiología del Instituto Nacional de Salud recibió la certificación para la incorporación de $S$. sonnei en la vigilancia molecular de esta red y se estableció su base de vigilancia molecular en Colombia.

El objetivo del presente estudio fue caracterizar molecularmente aislamientos de $S$. sonnei recuperados en el marco del programa de vigilancia por el laboratorio de la enfermedad diarreica aguda en Colombia desde 1997 hasta el 2013.

\section{Materiales y métodos}

\section{Tipo de estudio}

El estudio epidemiológico fue de tipo descriptivo y su objetivo fue caracterizar, mediante PFGE, una muestra aleatoria de aislamientos clínicos de $S$. sonnei en Colombia.

\section{Información epidemiológica}

Se estudiaron 102 de los 2.048 aislamientos de $S$. sonnei remitidos a la Red Nacional de Laboratorios entre 1997 y marzo del 2013 para iniciar la vigilancia genotípica de $S$. sonnei en el país mediante PFGE; la selección se hizo de acuerdo con el patrón de resistencia antimicrobiana, el origen de la muestra, los casos esporádicos y la relación con brotes. De los aislamientos estudiados, 43 procedían de Bogotá, 12 de Boyacá, 18 de Nariño, ocho de Antioquia, ocho del Valle, cinco de Cundinamarca, cuatro de Santander, dos de Risaralda, uno del Cesar y otro de Norte de Santander; 35 aislamientos se asociaron a diez brotes ocurridos en Bogotá $(n=3)$ y los departamentos de Boyacá $(n=8)$, Cundinamarca $(n=5)$, Nariño $(n=15)$ y Valle $(n=4)$, y 67 , a casos esporádicos.

El $48 \%(n=49)$ de los aislamientos correspondieron al sexo masculino y el $52 \%(n=53)$ al femenino; la distribución de los casos por edad se relacionó con casos entre los 0 y los 5 años ( $n=41,40,2 \%)$, entre los 6 y los 14 años $(n=39,38,2 \%)$ y a mayores de 14 ( $n=16,15,7 \%)$; el 5,9\% ( $n=6)$ de los aislamientos carecían del dato de la edad $(n=6$, $5,9 \%$ ). La mayoría de los aislamientos se recuperó de materia fecal ( $\mathrm{n}=95,93,1 \%)$, de hemocultivos $(n=3,2,9 \%)$, y de orina $(n=1,1,0 \%)$, y el $2,9 \%$ $(n=3)$ carecía de datos.

\section{Caracterización fenotípica}

Los aislamientos se habían confirmado previamente en el marco de las acciones de vigilancia del Laboratorio Nacional de Referencia de la Dirección de Redes en Salud Pública del Instituto Nacional de Salud, mediante pruebas bioquímicas
(19). La serotipificación se hizo con base en el esquema de Edwards y Ewing (20) mediante aglutinación en lámina usando los antisueros a, b y c (Eurobium).

Se determinó la sensibilidad microbiana a los siguientes antibióticos con el método de KirbyBauer: tetraciclina (TE) $(30 \mu \mathrm{g})$, cloranfenicol (C) $(30 \mu \mathrm{g})$, ácido nalidíxico (AN) $(30 \mu \mathrm{g})$, amoxicilinaácido clavulánico (AMC) $(10 \mu \mathrm{g})$ y ciprofloxacina (CIP) $(5 \mu \mathrm{g})$, y con el método de concentración inhibitoria mínima (MIC, MicroScan) se determinó la sensibilidad a los siguientes antibióticos: ampicilina (AMP) $(8-16 \mu \mathrm{g} / \mathrm{ml})$, cefotaxima (CTX) (2-32 $\mathrm{\mu g} / \mathrm{ml})$, ceftazidima (CAZ) (1-16 $\mu \mathrm{g} / \mathrm{ml})$, ciprofloxacina (CIP) (1-2 $\mu \mathrm{g} / \mathrm{ml})$, gentamicina (GM) (4-86 $\mu \mathrm{g} / \mathrm{ml})$, y trimetroprim-sulfametoxazol (SXT) $(2 / 38 \mu \mathrm{g} / \mathrm{ml})$ (Manual de procedimiento para Gram negativos de MicroScan $\left.{ }^{\circledR}\right)$; para ello se siguieron las recomendaciones y criterios del Clinical and Laboratory Standards Institute, 2013 (21).

\section{Caracterización genotípica}

La caracterización molecular de $S$. sonnei se hizo según el protocolo estandarizado por la red PulseNet International para la subtipificación molecular de $S$. sonnei utilizando la enzima de restricción primaria Xbal (Promega, USA), y la enzima de restricción secundaria BInl (Roche, USA), con el fin de confirmar la procedencia del $50 \%$ de los aislamientos recuperados de brotes. Se utilizó la cepa Salmonella Braenderup H9812 como marcador de peso molecular (18).

El análisis de los patrones de bandas generados mediante la PFGE se hizo con el programa Gel Compare $2 \AA$ V.4.0 (Applied Maths, Saint MartensLatem, Belgium). Se utilizó el coeficiente de Dice para comparar los patrones y establecer relaciones genéticas entre los aislamientos. La agrupación se basó en el algoritmo del método de pares de grupos no ponderados, usando la media aritmética (Unweighted Pair Group Method with Arithmetic Mean, UPGMA), con una tolerancia en la posición de las bandas de 1,5\% y una optimización de $1,5 \%$. La definición de las categorías de las relaciones genéticas entre los aislamientos, se hizo con base en los criterios de Tenover (22).

\section{Análisis de los datos}

Los datos se tabularon con el programa Microsoft Excel $^{\mathrm{TM}}$ y se analizaron con el paquete estadístico Epi Info ${ }^{\mathrm{TM}}$, versión 7.0; las diferencias estadísticamente significativas se evaluaron mediante la prueba de ji al cuadrado con un nivel de confianza 
del $95 \%$ para el sexo, la edad y el perfil de resistencia antimicrobiana según los resultados de la agrupación obtenida mediante PFGE-Xbal.

\section{Resultados}

\section{Análisis genotípico}

Se identificaron 42 patrones mediante PFGEXbal, los cuales se describen en la figura 1. Los patrones más frecuentes fueron COINJ6X01. 0017 ( $n=18 ; 17,64 \%)$, COINJ16X01.0004(n=10; $9,8 \%)$, COINJ16X01.0022 ( $n=6 ; 5,88 \%)$, y COINJ16X01.0031(n=5; 4,91\%) (figura 1).
Los patrones de PFGE obtenidos y la relación con el origen geográfico de los aislamientos de $S$. sonnei estudiados, se presentan en el cuadro $1, y$ los patrones más frecuentes y su distribución por grupos de edad y tipo de muestra, en el cuadro 2.

\section{Análisis del agrupamiento de los aislamientos mediante UPGMA}

En el dendrograma obtenido a partir de los patrones de PFGE con la enzima Xbal se establecieron tres grupos principales, considerando un índice de similitud del $70 \%$. El grupo I presentó $73,3 \%$ de similitud, el grupo II, $81,97 \%$, y el grupo III, $70 \%$. El

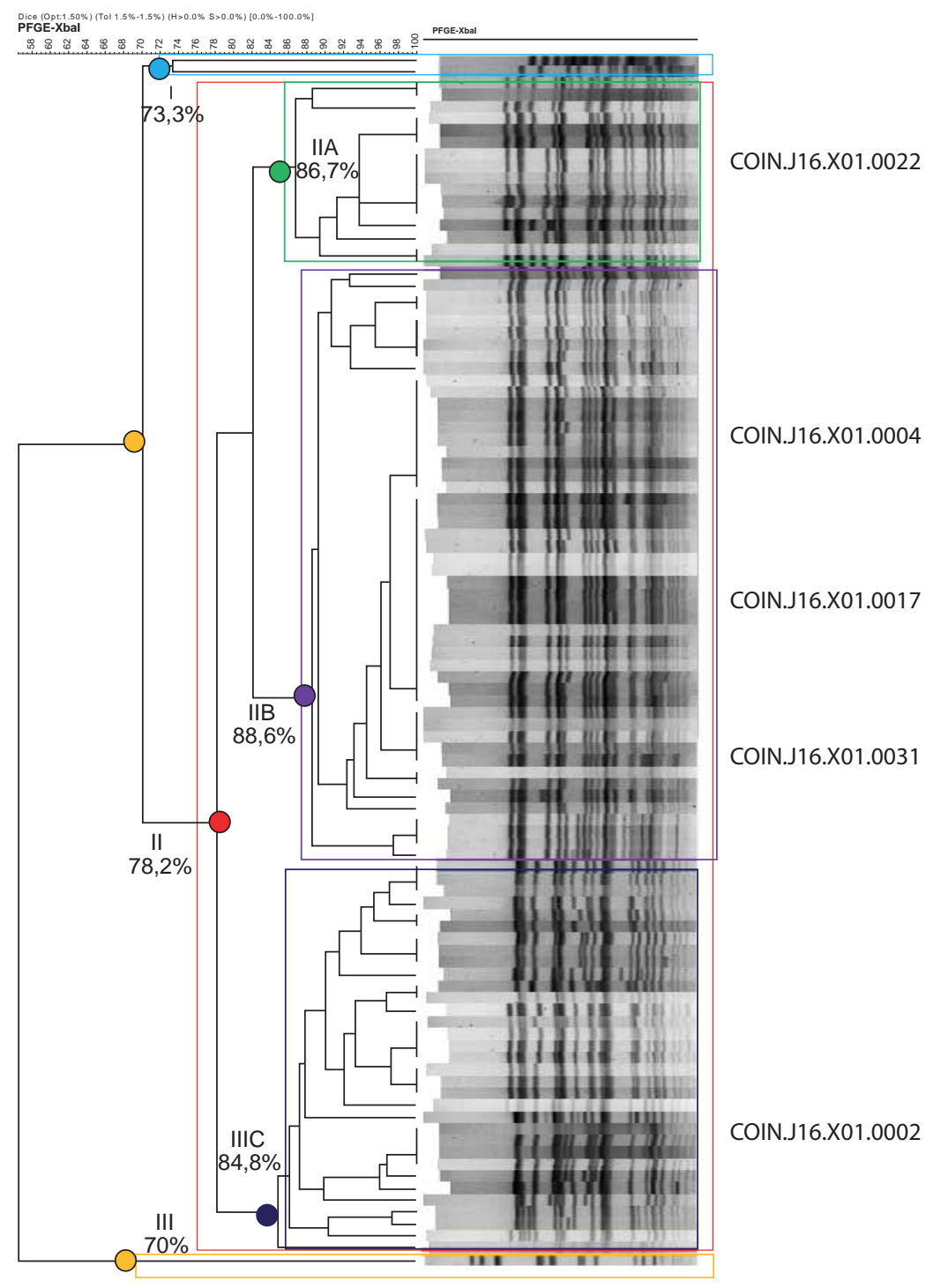

Figura 1. Dendrograma de la relación genética de los 42 patrones de PFGE-Xbal para los 102 aislamientos de S. sonnei. Programa GelCompar II, versión 4.0, porcentaje de tolerancia y optimización 1,5. Grupos principales (I-III); a la derecha de la figura se indican los patrones con más de tres aislamientos: COINJ16X01.0017 ( $n=18 ; 17,6 \%)$, COINJ16X01.0004 ( $n=10 ; 9,8 \%)$, COINJ16X01.0022 $(n=6 ; 5,9 \%)$, COINJ16X01.0031 ( $n=5 ; 4,9 \%)$. El 61,8\% ( $n=63)$ se agrupó en 38 patrones. El código de los patrones corresponde a CO: Colombia, IN: Instituto Nacional de Salud, J16: Shigella sonnei, X01: enzima Xbal. 
Cuadro 1. Distribución de los patrones de PFGE-Xbal de los aislamientos de Shigella sonnei por entidad territorial

\begin{tabular}{|c|c|c|c|c|c|c|c|c|c|c|c|}
\hline \multirow[t]{2}{*}{ Patrones } & \multicolumn{11}{|c|}{ Origen geográfico } \\
\hline & Bogotá & Nariño & Boyacá & Valle & Santander & Antioquia & $\begin{array}{l}\text { Norte de } \\
\text { Santander }\end{array}$ & Cesar & Risaralda & Cundinamarca & n (\%) \\
\hline COINJ16X01.0017 & 7 & 7 & 2 & 2 & & & & & & & $18(17,6)$ \\
\hline COINJ16X01.0004 & 5 & & 1 & 1 & 3 & & & & & & $10(9,8)$ \\
\hline COINJ16X01.0022 & 4 & & & & & 1 & 1 & & & & $6(5,9)$ \\
\hline COINJ16X01.0031 & 2 & 3 & & & & & & & & & $5(4,9)$ \\
\hline Otros patrones (38) & 25 & 8 & 9 & 5 & 1 & 7 & & 1 & 2 & 5 & $63(61,8)$ \\
\hline Total & 43 & 18 & 12 & 8 & 4 & 8 & 1 & 1 & 2 & 5 & 102 \\
\hline
\end{tabular}

Cuadro 2. Patrones PFGE-Xbal de los aislamientos de Shigella sonnei estudiados por grupo de edad y por tipo de muestra

\begin{tabular}{|c|c|c|c|c|c|c|c|c|c|c|c|c|}
\hline $\begin{array}{l}\text { Patrones } \\
\text { PFGE Xbal }\end{array}$ & COINJ16X01 & 0,0004 & 0,0006 & 0,0011 & 0,0012 & 0,0017 & 0,0022 & 0,0023 & 0,0029 & 0,0031 & $\begin{array}{c}\text { Otros } \\
\text { patrones }\end{array}$ & Total \\
\hline & & $\mathrm{n}(\%)$ & n (\%) & n (\%) & n (\%) & n (\%) & n (\%) & n (\%) & n (\%) & n (\%) & n (\%) & \\
\hline \multirow{4}{*}{$\begin{array}{l}\text { Grupos de } \\
\text { edad (años) }\end{array}$} & 0 a 5 & $5(12,2)$ & & & & $8 \quad(19,5)$ & & & $3(7,3)$ & & $25(61)$ & 41 \\
\hline & 6 a 14 & $5(12,8)$ & & $3(7,7)$ & & $3 \quad(7,7)$ & & & & & $28(71,8)$ & 39 \\
\hline & $>14$ & & & & & $3(18,8)$ & & $3(18,8)$ & & $4(25)$ & $6(37,5)$ & 16 \\
\hline & Total & 10 & & 3 & & 14 & & 3 & 3 & 4 & 59 & 96 \\
\hline \multirow{4}{*}{$\begin{array}{l}\text { Tipo de } \\
\text { muestra }\end{array}$} & Materia fecal & $10(10,5)$ & & & & $15(16,8)$ & $6(6,3)$ & & & $5(5,3)$ & $58(61,1)$ & 94 \\
\hline & Hemocultivo & & $1(33,3)$ & & $1(33,3)$ & & & & $1(33,3)$ & & & 3 \\
\hline & Orina & & & & & $1(100)$ & & & & & & 1 \\
\hline & Total & 10 & 1 & & 1 & 16 & 6 & & 1 & 5 & 58 & 98 \\
\hline
\end{tabular}

Los seis aislamientos faltantes por grupo de edad y los tres aislamientos faltantes por tipo de muestra, no tenían dato. Códigos de PFGE para denominar los patrones de Shigella sonnei: CO: Colombia, IN: Instituto Nacional de Salud, J16: Shigella sonnei, X01: enzima Xbal

grupo Il se dividió en tres subgrupos: en el subgrupo IIA, con una similitud de $86,7 \%$, se relacionaron cuatro grupos con aislamientos genéticamente indistinguibles (100\% de similitud) y tres grupos presentaron patrones únicos diferentes; en el subgrupo IIB, con $88,6 \%$ de similitud, se agruparon siete grupos de aislamientos genéticamente indistinguibles (100\% de similitud), y seis grupos presentaron patrones únicos, y el subgrupo IIC, con $84,8 \%$ de similitud, incluyó siete grupos que relacionaron aislamientos genéticamente indistinguibles (100\% de similitud) y 12 grupos con patrones únicos (figura 1).

Con la enzima BInl se identificaron 11 patrones de PFGE de los diez brotes $(n=35)$ estudiados entre 2002 y 2013. Se seleccionó el $50 \%$ de los aislamientos provenientes de los brotes remitidos al Grupo de Microbiología ( $n=22)$ para la confirmación mediante la enzima BInl. Se establecieron dos grupos principales: el grupo i presentó $79,5 \%$ de similitud y el grupo ii, $97,5 \%$. El grupo i se dividió en dos subgrupos: ia, con 90,2\% de similitud, e ib, con $81,1 \%$ (figura 2 , cuadro 3 ).

\section{Sensibilidad microbiana}

De los 102 aislamientos seleccionados, el 63,8\% $(\mathrm{n}=65)$ presentó multirresistencia a tres o más familias de antibióticos; el patrón de multirresistencia más frecuente para tres familias de antibióticos correspondió a TE-C-AMC $(n=9$; $8,8 \%$ ), en tanto que el patrón TE-C-SXT-AMPAMC $(n=19 ; 18,9 \%)$, fue el más frecuentemente relacionado con cuatro familias de antibióticos. El $26,4 \%(n=27)$ de los aislamientos presentaron resistencia a una o dos familias de antibióticos y el 9,8\% $(n=10)$ de los restantes fueron sensibles. La relación del perfil de resistencia y el patrón de PFGE se presentan en el cuadro 4.

La correlación de los patrones de PFGE-Xbal y el patrón de resistencia a agentes antimicrobianos, reveló que el patrón más frecuente fue el COINJ16X01.0017 (cuadro 5), el cual presentó el patrón de resistencia TE-C-SXT-AMP-AMC en 10 aislamientos (52,6\%); el patrón de PFGE-Xbal COINJ16X01.0004 presentó dos patrones de resistencia más comunes: TE-CNA-AMP-AMC y TE-C-SXT-AMP-AMC en dos aislamientos (20\%), mientras que el patrón de PFGE-Xbal COINJ16X01.0031 presentó el patrón multirresistente TE-C-AMC en tres aislamientos $(60 \%)$. No se identificó una asociación entre los patrones de resistencia y los perfiles de PFGE con respecto a la edad ni al origen geográfico. 


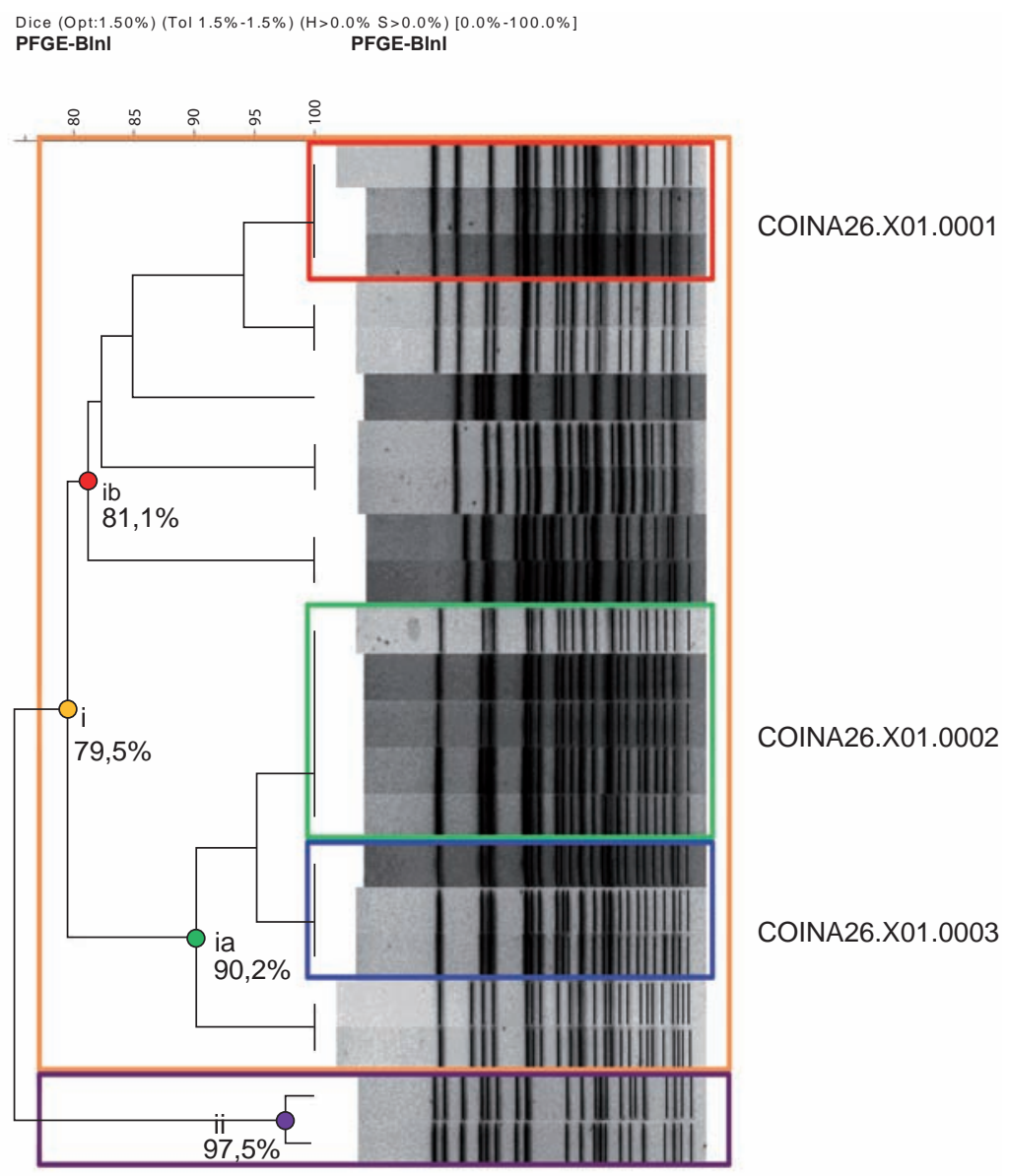

Figura 2. Dendrograma de la relación genética de los patrones de PFGE- BInl para el $50 \%(n=22)$ de los aislamientos de $S$. sonnei asociados a brotes. Programa GelCompar II, versión 4.0, porcentaje de tolerancia y optimización 1,5. Grupos principales: el grupo i, con el $79,5 \%$ de similitud, y el grupo ii, con un $97,5 \%$ de similitud (i-ii). A la derecha de la figura se indican los patrones frecuentes: COINJ16A26.0003 $(n=3)$, COINJ16A26.0002 $(n=5)$ y COINJ16A26.0001 $(n=3)$.

\section{Análisis estadístico}

El análisis estadístico se basó en la agrupación obtenida mediante PFGE-Xbal en el grupo II (IIA, IIB y IIC); los grupos I y III se excluyeron del análisis debido al número de aislamientos asociados.

En el análisis del grupo II (IIA, IIB y IIC) se incluyeron las variables de edad, sexo y patrón de resistencia antimicrobiana en cada subgrupo. Al considerar las variables de edad y de sexo, no se encontró asociación estadísticamente significativa entre el patrón de PFGE-Xbal y dichas variables en ninguno de los subgrupos (IIA, IIB y IIC).

Con los datos de la muestra, el patrón de PFGEXbal se relacionó con el patrón de multirresistencia en los subgrupos IIA y IIB (ji al cuadrado: $p=0,000$ ), y no se encontró relación estadísticamente significativa entre el patrón de PFGE-Xbal y el patrón de resistencia (cuadro 6).

\section{Discusión}

La importancia de $S$. sonnei en Colombia se debe a su frecuente notificación (53,5\%) y a su asociación con los brotes (datos sin publicar, Grupo de Microbiología, diciembre de 2013). En este estudio se determinó la relación genotípica de 102 de 2.048 aislamientos colombianos de $S$. sonnei recuperados entre 1997 y 2013 mediante PFGE, y se identificaron 42 patrones.

La técnica de PFGE ha demostrado ser una herramienta útil para la discriminación de aislamientos y la identificación clonal de linajes en varias especies bacterianas debido a que ofrece una visión general de la arquitectura de su cromosoma. Mediante el análisis de recombinación de ADN, Karaolis estableció que $S$. sonnei es genéticamente homogénea, con bajos porcentajes de recombinación a lo largo del tiempo, es decir que es una especie muy conservada y clonal (23). 
Cuadro 3. Distribución de los patrones de PFGE-Xbal de los aislamientos de Shigella sonnei estudiados asociados a brotes por departamentos

\begin{tabular}{|c|c|c|c|c|c|c|}
\hline $\begin{array}{l}\text { Fecha (semana } \\
\text { epidemiológica) }\end{array}$ & $\begin{array}{l}\text { Entidades } \\
\text { territoriales }\end{array}$ & $\begin{array}{c}\text { Total } \\
\text { personas } \\
\text { implicadas } \\
\text { (n) }\end{array}$ & $\begin{array}{l}\text { Aislamientos } \\
\text { estudiados } \\
\text { por PFGE (n) }\end{array}$ & Patrón PFGE - Xbal & Patrón PFGE - BInl & Patrón de resistencia \\
\hline $11 / 07 / 2002$ & Cundinamarca & Sd & 5 & $\begin{array}{l}\text { COIN02J16X01.0002 } \\
(n=4) \\
\text { COIN02J16X01.0041 } \\
(n=1)\end{array}$ & COIN02A26X01.0001 & TE-SXT-AMP \\
\hline $31 / 07 / 2008$ & $\begin{array}{l}\text { Cundinamarca/ } \\
\text { Bogotá }\end{array}$ & $\mathrm{Sd}$ & 3 & $\begin{array}{l}\text { COIN08J16X01.0013 } \\
(n=2) \\
\text { COIN08J16X01.0012 } \\
(n=1)\end{array}$ & COIN08A26X01.0004 & TE-SXT \\
\hline 23/09/2008 & Boyacá & Sd & 3 & $\begin{array}{l}\text { COIN08J16X01.0014 } \\
(n=1) \\
\text { COIN08J16X01.0015 } \\
(n=1) \\
\text { COIN08J16X01.0016 } \\
(n=1)\end{array}$ & $\begin{array}{l}\text { COIN08A26X01.0005 }(n=1) \\
\text { COIN08A26X01.0003 }(n=1)\end{array}$ & TE-SXT \\
\hline 28/10/2008 & Nariño & Sd & 2 & $\begin{array}{l}\text { COIN08J16X01.0017 } \\
(\mathrm{n}=2)\end{array}$ & COIN08A26X01.0002 & $\begin{array}{l}\text { TE-C-SXT-AMP-AMC } \\
\text { TE-C-AMP-CTX-AMC }\end{array}$ \\
\hline $01 / 12 / 2008$ & Nariño/La Cruz & 163 & 5 & $\begin{array}{l}\text { COIN08J16X01.0017 } \\
(n=4) \\
\text { COIN08J16X01.0027 } \\
(n=1)\end{array}$ & COIN08A26X01.0002 & $\begin{array}{l}\text { TE-C-SXT-AMP-AMC } \\
\text { AMC }\end{array}$ \\
\hline 22/07/2009 & $\begin{array}{l}\text { Boyacá/Santa } \\
\text { Sofía }\end{array}$ & 8 & 3 & $\begin{array}{l}\text { COIN09J16X01.0020 } \\
(n=2) \\
\text { COIN09J16X01.0021 } \\
(n=1)\end{array}$ & $\begin{array}{l}\text { COIN09A26X01.0007 } \\
\text { COIN09A26X01.0008 }\end{array}$ & TE-SXT \\
\hline 15/09/2009 & $\begin{array}{l}\text { Boyacá/ } \\
\text { Sotaquirá }\end{array}$ & 12 & 2 & $\begin{array}{l}\text { COIN09J16X01.0023 } \\
(\mathrm{n}=2)\end{array}$ & COIN09A26X01.0006 & TE-SXT / TE-SXT-AMP \\
\hline $30 / 08 / 2012$ & Nariño & 22 & 4 & $\begin{array}{l}\text { COIN12J16X01.0031 } \\
(n=3) \\
\text { COIN12J16X01.0032 } \\
(n=1)\end{array}$ & COIN12A26X01.0003 & TE-C-AMC \\
\hline $24 / 09 / 2012$ & $\begin{array}{l}\text { Nariño/ } \\
\text { Gualmatán }\end{array}$ & 6 & 4 & $\begin{array}{l}\text { COIN12J16X01.0029 } \\
(n=3) \\
\text { COIN12J16X01.0033 } \\
(n=1)\end{array}$ & COIN12A26X01.0010 & $\begin{array}{l}\text { TE-C-AMC } \\
\text { TE }\end{array}$ \\
\hline 07/02/2013 & Valle & 18 & 4 & $\begin{array}{l}\text { COIN13J16X01.0033 } \\
(n=2) \\
\text { COIN13J16X01.0039 } \\
(n=1) \\
\text { COIN13J16X01.0004 } \\
(n=1)\end{array}$ & COIN12A26X01.0011 & TE-C-AMP \\
\hline
\end{tabular}

Sd: $\sin$ dato

Recientemente, Holt encontró evidencia sólida del agrupamiento regional de $S$. sonnei, lo que indica una asociación geográfica (24).

En este estudio, la agrupación de los 102 aislamientos de $S$. sonnei mediante PFGE-Xbal permitió determinar 42 patrones en tres grupos (I-III) con alrededor de $70 \%$ de similitud. Algunos hallazgos reportados previamente confirman la naturaleza clonal de S. sonnei. Mammina, et al. (2005), relacionaron aislamientos de regiones distantes del sur de Italia con casos importados de África mediante PFGE, estableciendo dos grupos en 64 aislamientos: $A(n=13)$ y $B(n=51)$; en el grupo $A$ los patrones fueron indistinguibles y el grupo $B$ se subdividió en tres grupos diferentes (B1, B2 y B3), que diferían entre ellos en menos de tres bandas, por lo que se consideraron como de relación próxima (25). En un análisis mediante PFGE, Pazhani asignó un patrón idéntico a 11 de 17 aislamientos de S. sonnei, en tanto que dos aislamientos se agruparon como cercanamente relacionados con este grupo, con diferencias en dos o tres bandas, y cuatro aislamientos no se relacionaron genéticamente (11). Pichel, et al., agruparon una muestra de $S$. sonnei en cuatro grupos principales (A-D) mediante PFGE-Xbal y otros cuatro (I-IV) mediante PFGE-BInl, y determinaron 21 patrones de casos esporádicos y cinco patrones asociados a brotes en 56 aislamientos (16). 
Cuadro 4. Relación entre el patrón de PFGE y el perfil de resistencia o multirresistencia por año, número de aislamientos y departamentos relacionados

\begin{tabular}{lcccc}
\hline Año & N $^{\circ}$ aislamientos & Patrones de PFGE-Xbal & $\begin{array}{c}\text { Perfil de resistencia/ } \\
\text { multirresistencia }\end{array}$ & Entidad territorial \\
\hline $1997-2011$ & 61 & 30 & $3 / 10$ & $\begin{array}{l}\text { Antioquia, Bogotá, Boyacá, Cesar, } \\
\text { Cundinamarca, Nariño, Risaralda, } \\
\text { Santander, Valle }\end{array}$ \\
2012 & 11 & & Bogotá, Boyacá, Nariño \\
2013 & 30 & 7 & $2 / 3$ & Antioquia, Bogotá, Boyacá, \\
& & 16 & Norte de Santander, Valle & \\
\hline
\end{tabular}

Cuadro 5. Análisis comparativo de los patrones de PFGE-Xbal y los patrones de resistencia antimicrobiana más frecuentes en los aislamientos de Shigella Sonnei estudiados

\begin{tabular}{|c|c|c|c|c|c|c|}
\hline \multicolumn{7}{|c|}{ Patrón de resistencia antimicrobiana } \\
\hline Patrones de PFGE- Xbal & TE-C-AMC & $\begin{array}{l}\text { TE-C-NA- } \\
\text { AMP-AMC }\end{array}$ & $\begin{array}{l}\text { TE-C-SXT- } \\
\text { AMP-AMC }\end{array}$ & TE-SXT & TE-SXT-AMP & $\begin{array}{l}\text { Total } \\
\text { n (\%) }\end{array}$ \\
\hline COINJ16X01.0017 & & 2 & 10 & & & $12(20,6)$ \\
\hline COINJ16X01.0004 & 1 & 2 & 2 & & & $5 \quad(8,6)$ \\
\hline COINJ16X01.0002 & & & & & 4 & $4 \quad(6,8)$ \\
\hline COINJ16X01.0031 & 3 & 1 & & & & $4 \quad(6,8)$ \\
\hline COINJ16X01.0009 & & 1 & 2 & & & $3 \quad(5,17)$ \\
\hline COINJ16X01.0013 & & & & 3 & & $3 \quad(5,17)$ \\
\hline COINJ16X01.0023 & & & & 2 & 1 & $3(5,17)$ \\
\hline COINJ16X01.0029 & 3 & & & & & $3 \quad(5,17)$ \\
\hline COINJ16X01.0012 & & & & 2 & & $2 \quad(3,4)$ \\
\hline COINJ16X01.0018 & & & 2 & & & $2 \quad(3,4)$ \\
\hline COINJ16X01.0020 & & & & 2 & & $2 \quad(3,4)$ \\
\hline COINJ16X01.0037 & & & & 2 & & $2 \quad(3,4)$ \\
\hline COINJ16X01.0032, 0035 & $1^{\mathrm{a}}$ & & & & & $2 \quad(3,4)^{\mathrm{b}}$ \\
\hline COINJ16X01.0006,0011, 0041 & & & & & $1^{\mathrm{a}}$ & $3 \quad(5,17)^{\mathrm{b}}$ \\
\hline COINJ16X01.0025,0028,0034 & & & $1^{\mathrm{a}}$ & & & $3 \quad(5,17)^{\mathrm{b}}$ \\
\hline COINJ16X01.0014,0015,0016,0021, 0040 & & & & $1^{a}$ & & $5 \quad(8,6)^{b}$ \\
\hline Total general & 9 & 6 & 19 & 16 & 8 & $58(100 \%)$ \\
\hline
\end{tabular}

En este estudio se encontró que en algunos patrones asociados con brotes se incluyeron aislamientos esporádicos no relacionados. El patrón COINJ16X01.0017 colombiano se relacionó con cinco aislamientos de un brote en Nariño ocurrido en el 2008 y con 14 aislamientos no relacionados, dos del 2004 provenientes de Bogotá y Valle, y 12 de los años 2008, 2010, 2011 y 2013 provenientes de Nariño, Bogotá, Boyacá y Valle, lo cual indica la dispersión de estos patrones en otros departamentos del país.

En el estudio de Pichel, los brotes se agruparon en cuatro grupos principales y algunos aislamientos de casos esporádicos procedentes de áreas muy distantes se relacionaron mediante PFGE con los aislamientos de los brotes (16). En el análisis de los 35 aislamientos relacionados con diez brotes según los criterios de Tenover, la relación establecida permitió considerar que en dos de los brotes (Boyacá, 2009, y Nariño, 2008), se presentó un patrón de PFGE indistinguible, y en los otros ocho se encontraron patrones con una relación muy cercana, con cambios en dos o tres bandas de diferencia (22).

La resistencia a varios antimicrobianos en Shigella spp. se ha incrementado en los últimos años como viene reportándose en diferentes regiones del mundo $(8-11,26)$, y se asocia principalmente a mecanismos de resistencia relacionados con la presencia de plásmidos, integrones de clase 1-2 y betalactamasas (27-30).

En los aislamientos colombianos de $S$. sonnei estudiados la sensibilidad a todos los antibióticos evaluados fue de 9,8 \% (10/102) entre 2004 y 2011; la multirresistencia antimicrobiana en estos 
Cuadro 6. Conjunto de análisis basados en la agrupación obtenida mediante PFGE-Xbal del grupo II (IIA, IIB y IIC)

\begin{tabular}{|c|c|c|c|}
\hline Grupo II & Variable & Categoría & $p$ \\
\hline \multirow[t]{6}{*}{ A } & Sexo & $\begin{array}{l}\text { Masculino } \\
\text { Femenino }\end{array}$ & 0,497 \\
\hline & Edad (años) & $0-4$ & 0,296 \\
\hline & & $5-15$ & 0,139 \\
\hline & & $>16$ & 0,584 \\
\hline & Patrón de resistencia & Resistente & 0,492 \\
\hline & antimicrobiano & Multirresistente & $0,000^{*}$ \\
\hline \multirow[t]{6}{*}{ B } & Sexo & $\begin{array}{l}\text { Masculino } \\
\text { Femenino }\end{array}$ & 0,367 \\
\hline & Edad (años) & $0-4$ & 0,928 \\
\hline & & $5-15$ & 0,581 \\
\hline & & $>16$ & 0,551 \\
\hline & Patrón de resistencia & Resistente & 0,551 \\
\hline & antimicrobiano & Multirresistente & $0,000^{*}$ \\
\hline \multirow[t]{7}{*}{ C } & Sexo & Masculino & 0,670 \\
\hline & & Femenino & \\
\hline & Edad (años) & $0-4$ & 0,459 \\
\hline & & $5-15$ & 0,549 \\
\hline & & $>16$ & 0,846 \\
\hline & Patrón de resistencia & Resistente & 0,627 \\
\hline & antimicrobiano & Multirresistente & 0,179 \\
\hline
\end{tabular}

*Prueba estadísticamente significativa

aislamientos se ha observado desde 1998, cuando se encontró el patrón de PFGE COIN98J16X01. 0001 en un aislamiento de un paciente de dos años procedente de Risaralda. La resistencia a cefalosporinas de tercera generación solo se presentó para la cefotaxima (CTX) en 2,9\% $(n=3)$ de los aislamientos en los años 1998, 2005, 2008, y a la ceftazidima (CAZ) en $0,98 \%$ de ellos $(n=1)$ en el 2005.

La resistencia al ácido nalidíxico se confirmó en 17 aislamientos entre el 2003 y el 2013, 12 de los cuales presentaban alta resistencia $y$, cinco, resistencia intermedia; debe recordarse que el ácido nalidíxico se considera un factor predictor de una disminución de la sensibilidad a la ciprofloxacina y a las fluoroquinolonas, línea de antibióticos recomendada por la OMS para el tratamiento de infecciones por Shigella spp. (7). Se observó la resistencia a la ciprofloxacina en un aislamiento del 2012 procedente de un paciente de 7 años en Bogotá, el cual presentaba el patrón COIN12J16X01.0003, lo que resalta la importancia de la vigilancia antimicrobiana de estos mecanismos, ya que esta tendencia podría darse en los aislamientos asociados con infecciones por S. sonnei en el país.

La resistencia al ácido nalidíxico y a la ciprofloxacina en Asia y África, se ha incrementado progresivamente entre el 2007 y el 2009, alcanzando
64,5 y $29,1 \%$, respectivamente. En Europa y América se siguen registrando niveles bajos de resistencia ( $<5$ y $<1 \%$, respectivamente) (31).

La relación obtenida mediante la agrupación permite encontrar patrones y relaciones significativas. Reukit, et al., reportaron en la agrupación clonal de aislamientos de $S$. sonnei cuatro grupos principales: en el grupo 2, representado por aislamientos con un alto porcentaje de similitud (95,9 a 99,7\%) y un mismo patrón de multirresistencia, se pudo establecer la relación entre los aislamientos estudiados del subgrupo clonal IIA-IIB con el perfil de multirresistencia, lo cual permitió establecer una relación estadísticamente significativa que podría indicar su relación con los perfiles de multirresistencia (32).

Khan, et al., demostraron recientemente que las manifestaciones extraintestinales de la shigelosis no se relacionaban exclusivamente con $S$. dysenteriae de tipo 1, como se había considerado hasta entonces (33). El espectro de las complicaciones intestinales y extraintestinales graves que producen todas las especies de Shigella spp. hace de esta una enfermedad difícil de tratar una vez que las complicaciones se han desarrollado.

Las infecciones extraintestinales como las bacteriemias no son comunes; se considera que la infección neonatal por $S$. sonnei produce gran morbilidad y mortalidad, y complicaciones como la enterocolitis y la septicemia (34).

Se analizaron aislamientos de hemocultivos con infección por $S$. sonnei de origen extraintestinal $(2,9 \%)$, uno de los cuales se había aislado en un recién nacido procedente de Bogotá y presentaba el patrón COIN11J16X01.0029, con resistencia a TE; el segundo aislamiento correspondía a un paciente de 4 años con síndrome febril procedente de Antioquia, el cual presentó el patrón COIN05J16X01.0006 con multirresistencia a SXTAMP-TE, y el tercer caso, un adulto de 35 años positivo para HIV procedente de Risaralda, presentó el patrón de PFGE COIN09J16X01.0012 con resistencia a SXT-TE, lo cual resalta la importancia de la vigilancia de esta enfermedad, ya que puede afectar a grupos de población vulnerable con manifestaciones clínicas más graves.

En este estudio se estableció la base de datos de los patrones electroforéticos de aislamientos de $S$. sonnei mediante PFGE, los cuales serán incorporados en la base nacional de datos. Con ello se contribuye a la investigación de brotes y 
casos esporádicos en Colombia y, además, la información de dicha base alimenta la base de datos regional del Centro de Referencia Regional de PulseNet América Latina y Caribe, que opera en el Instituto de Enfermedades Infecciosas, ANLIS, "Dr. Carlos G. Malbrán" en Argentina. Esta información se comparte con los países miembro de la red PulseNet en caso de alertas internacionales de brotes y para la notificación de los patrones de circulación común en la región que pueden persistir en el tiempo.

Al comparar los patrones de PFGE colombianos con la base regional de datos, se encontraron patrones detectados en otros países de Latinoamérica. Es el caso de los patrones COINJ16X01.0017 y COINJ16X01.0004, los cuales se relacionaron con algunos de Perú (Laboratorio de Referencia Nacional de Enteropatógenos, Centro Nacional de Salud Pública, Instituto Nacional de Salud, Lima LRN- INS, Perú, http://www.ins.gob.pe/portal/homecnsp/), así como del patrón COINJ16X01.0022, relacionado con un patrón de PFGE de Costa Rica (Centro Nacional de Referencia de Bacteriología, INCIENSA, Costa Rica, http://www.inciensa.sa.cr/ inciensa/centros_referencia/bacteriologia.aspx), el patrón COINJ16X01.0003 relacionado con uno de Chile (Laboratorio de Genética Molecular y Sección de Bacteriología, Instituto Nacional de Salud Pública de Chile, http://www.ispch.cl/vigilanciabacteriologia-0), el patrón COINJ16X01.0028 relacionado con un patrón de Argentina (Red de Diarreas y Patógenos Bacterianos de Transmisión Alimentaria, Servicio de Fisiopatogenia y Enterobacterias, Antígenos y Antisueros, Instituto Nacional de Producción (INPB)-INEI-ANLIS, http://www.anlis. gov.ar/cnrl/?page_id=117), y el patrón COINJ16X01. 0021, presente en los años 2009 y 2013, el cual se relacionó con un patrón de Brasil (Centro de Bacteriología, Instituto Adolfo Lutz, Brasil) (datos compartidos por la Red PulseNet Regional América Latina y Caribe, Instituto de Enfermedades Infecciosas, ANLIS, "Dr. Carlos G. Malbrán”).

Estos patrones electroforéticos reportados en otros países sin vinculación epidemiológica aparente pueden estar asociados con viajes de personas dentro de estos países o a zonas fronterizas, así como con fuentes comunes de infección, con el potencial de diseminación de diferentes clones que persisten durante largo tiempo y la transmisión de persona a persona de una cepa particular con cambios genéticos menores a lo largo de un extenso período, o con una combinación de todos estos eventos $(35,36)$.
Al considerar factores como el incremento en la frecuencia de $S$. sonnei con resistencia antimicrobiana a nivel global y la capacidad de dispersión de los aislamientos asociados con morbilidad y mortalidad, se entiende por qué este patógeno causante de la enfermedad diarreica aguda es un problema emergente de salud pública que requiere seguimiento microbiológico y epidemiológico, así como el fortalecimiento del sistema nacional de vigilancia de patógenos entéricos.

\section{Agradecimientos}

A los laboratorios de salud pública de Colombia. A la Dra. Elizabeth Castañeda, Investigadora Emérita del Instituto Nacional de Salud, por su revisión crítica y sus aportes al manuscrito. A la red PulseNet América Latina y Caribe por permitir la comparación con la base de datos regional. A María Luz Zamudio del Instituto Nacional de Salud de Perú. A Anabella della Gaspera, Josefina Campos e Isabel Chinen del INEI-ANLIS de Argentina. A Francisco Duarte del INCIENSA de Costa Rica. A Jorge Fernández del Instituto Nacional de Salud Pública de Chile. A Ana Terezinha Tavechio, Sueli Aparecida Fernandes y Tania Mara Ibelli Vaz del Instituto Adolfo Lutz de Brasil. A Enrique Pérez Gutiérrez, asesor senior en enfermedades transmitidas por alimentos y zoonosis del Departamento de Enfermedades Transmisibles y Análisis de la Salud de la OPS-OMS.

\section{Conflicto de intereses}

Las autoras declaran no tener conflicto de intereses.

\section{Financiación}

Grupo de Microbiología, Subdirección de Investigación Científica y Tecnológica y Subdirección de Red de Laboratorios, Instituto Nacional de Salud. Programa Jóvenes Investigadores, Colciencias, convocatoria 525-2011.

\section{Referencias}

1. Bhattacharya D, Sugunan AP, Bhattacharjee $\mathbf{H}$, Thamizhmani R, Sayi DS, Thanase K, et al. Antimicrobial resistance in Shigella -rapid increase \& widening of spectrum in Andaman Islands. Indian J Med Res. 2012;135:365-70.

2. Shakoor S, Zaidi AK, Hasan R. Tropical bacterial gastrointestinal infections. Infect Dis Clin North Am. 2012;26:43753. http://dx.doi.org/10.1016/j.idc.2012.02.002

3. World Health Organization. Future needs and directions for Shigella vaccines. Wkly Epidemiol Rec. 2006;81:51-8.

4. Kotloff KL, Winickoff JP, Ivanoff B, Clemens JD, Swerdlow DL, Sansonetti P, et al. Global burden of Shigella infections: Implications for vaccine development and implementation of control strategies. Bull World Health Organ. 1999 77:651-66. 
5. Mandal J, Emelda J, Parija SC. The recent trends of Shigellosis: A JIPMER perspective. J Clin Diagn Res. 2012;6:1474-7. http://dx.doi.org/10.7860/JCDR/2012/4157. 2536

6. Agtini MD, Soeharno R, Lesmana $M$, Punjabi $\mathbf{N H}$, Simanjuntak C, Wangsasaputra F, et al. The burden of diarrhoea, shigellosis, and cholera in North Jakarta, Indonesia: Findings from 24 months surveillance. BMC Infect Dis. 2005;20:5-89. http://dx.doi.org/10.1186/1471 2334-5-89

7. World Health Organization. Guidelines for the control of shigellosis, including epidemics due to Shigella dysenteriae type 1 . Fecha de consulta: 10 de abril de 2015. Disponible en: http://whqlibdoc.who.int/publications/2005/9241592330. pdf.

8. Kosek M, Yori PP, Pan WK, Olortegui MP, Gilman RH, Pérez $\mathbf{J}$, et al. The epidemiology of the highly endemic, multiple antibiotic-resistant shigellosis in children in the Peruvian Amazon. Pediatr. 2008;122:e541-9. http://dx.doi. org/10.1542/peds.2008-0458

9. Vinh H, Baker S, Campbell J, Hoang NV, Loan HT, Chinh MT, et al. The rapid emergence of third generation cephalosporin resistant Shigella spp. in southern Vietnam. J Med Microbiol. 2009;58:281-3. http://dx.doi.org/10.1099/ jmm.0.002949-0

10. Srinivasan $\mathbf{H}$, Baijayanthi $\mathbf{M}$, Raksha $\mathbf{Y}$. The magnitude of the drug resistant shigellosis: A report from Bangalore. Indian J Med Microbiol. 2009;27:358-60. http://dx.doi. org/10.4103/0255-0857.55460

11. Pazhani G, Niyogi S, Singh A, Sen B, Taneja N, Kundu $\mathbf{M}$, et al. The molecular characterization of the multidrug resistant Shigella spp. which were isolated from the epidemic and the endemic cases of shigellosis in India. $J$ Med Microbiol. 2008;57:856-63. http://dx.doi.org/10.1099/ jmm.0.2008/000521-0

12. von Seidlein L, Kim DR, Ali M, Lee H, Wang X, Thiem VD, et al. A multicentre study of Shigella diarrhoea in six Asian countries: Disease burden, clinical manifestations, and microbiology. J PLoS Med. 2006;3:e353. http://dx.doi. org/10.1371/journal.pmed.0030353

13. Vinh H, Nhu NT, Nga TV, Duy PT, Campbell JI, Hoang NV, et al. A changing picture of shigellosis in southern Vietnam: Shifting species dominance, antimicrobial susceptibility and clinical presentation. BMC Infect Dis. 2009;15:204. http://dx. doi.org/10.1186/1471-2334-9-204

14. Banga Singh K-K, Ojha SC, Deris ZZ, Rahman RA. A 9-year study of shigellosis in Northeast Malaysia: Antimicrobial susceptibility and shifting species dominance. Z Gesundh Wiss. 2011;19:231-6. http://dx.doi.org/10.1007/ s10389-010-0384-0

15. Das SK, Ahmed S, Ferdous F, Farzana FD, Chisti MJ, Leung DT, et al. Changing emergence of Shigella serogroups in Bangladesh: Observation from four different diarrheal disease hospitals. PLoS One. 2013;8:e62029. http://dx.doi.org/10.1371/journal.pone.0062029

16. Pichel M, González S, Terragno R, Mulki J, Gentile A, Kremer C, et al. Short report: Analysis of clonal relationship among Shigella sonnei isolates circulating in Argentina. Epidemiol Infect. 2007;135:681-7. http://dx.doi.org/10.1017/ S0950268806007230
17. Instituto Nacional de Salud. Vigilancia de los patrones de Shigella spp. Fecha de consulta: 10 de abril de 2015. Disponible en: http://www.ins.gov.co/tramites-yservicios/examenes-de-inter\%C3\%A9s-en-salud-publica/ Microbiologa/Microbiolog\%C3\%ADa\%20Shi.pdf.

18. Ribot EM, Fair MA, Gautom R, Cameron DN, Hunter SB, Swaminathan B, et al. Standardization of pulsed-field gel electrophoresis protocols for the subtyping of Escherichia coli O157:H7, Salmonella, and Shigella for PulseNet. Foodborne Pathog Dis. 2006;3:59-67. http://dx.doi.org/10. 1089/fpd.2006.3.59

19. Bopp C, Brenner F, Fields P, Wells J, Strockbine N. Escherichia, Shigella, and Salmonella. En: Murray P, Baron E, Jorgensen J, Pfaller M, Yolken R, editors. Manual of Clinical Microbiology. 8th edition. Washington, D.C.; ASM Press; 2003. p. 654-71.

20. Edwards PR, Ewing WH. Identification of Enterobacteriaceae. 4th edition. New York: Elsevier; 1986. p. 137-72.

21. Clinical and Laboratory Standards Institute. Performance standards for antimicrobial susceptibility testing; Twentythird informational supplement. CLSI document M100. Wayne, Pennsylvania: CLSI; 2013.

22. Tenover FC, Arbeit RD, Goering RV, Mickelsen PA, Murray BE, Persing DH, et al. Interpreting chromosomal DNA restriction patterns produced by pulsed-field gel electrophoresis: Criteria for bacterial strain typing. J Clin Microbiol.1995;33:2233-9.

23. Karaolis DK, Lan R, Reeves PR. Sequence variation in Shigella sonnei (Sonnei), a pathogenic clone of Escherichia coli, over four continents and 41 years. J Clin Microbiol. 1994;32:796-802.

24. Holt K, Baker S, Weill F, Holmes E, Kitchen A, Yu J, et al. Shigella sonnei genome sequencing and phylogenetic analysis indicate recent global dissemination from Europe. Nat Genet. 2012;44:1056-9. http://dx.doi.org/10.1038/ng. 2369

25. Mammina C, Pontello M, Dal Vecchio A, Nastasi A, The S. sonnei Working Group. Identification of Shigella sonnei biotype $\mathrm{g}$ isolates carrying class 2 integrons in Italy (2001 to 2003). J Clin Microbiol. 2005;43:2467-70. http://dx.doi. org/10.1128/JCM.43.5.2467-2470.2005

26. Zaid MB, Estrada-García T, Campos FD, Chim R, Arjona $\mathbf{F}$, Leon $\mathbf{M}$, et al. Incidence, clinical presentation, and antimicrobial resistance trends in Salmonella and Shigella infections from children in Yucatán, México. Front Microbiol. 2013:4;288. http://dx.doi.org/10.3389/fmicb.2013.00288

27. Ahmed AM, Furuta K, Shimomura K, Kasama Y, Shimamoto T. Genetic characterization of multidrug resistance in Shigella spp. from Japan. J Med Microbiol. 2006;55:1685-91. http://dx.doi.org/10.1099/jmm.0.46725-0

28. Jin YH, Oh YH, Jung JH, Kim SJ, Kim JA, Han KY, et al. Antimicrobial resistance patterns and characterization of integrons of Shigella sonnei isolates in Seoul, 1999-2008. J Microbiol. 2010;48:236-42. http://dx.doi.org/10.1007/ s12275-010-9220-z

29. Ke X, Gu B, Pan S, Tong M. Epidemiology and molecular mechanism of integron-mediated antibiotic resistance in Shigella. Arch Microbiol. 2011;193:767-74. http://dx.doi. org/10.1007/s00203-011-0744-3 
30. Navia MM, Capitano L, Ruiz J, Vargas M, Urassa H, Schellemberg $D$, et al. Typing and characterization of mechanisms of resistance of Shigella spp. isolated from feces of children under 5 years of age from Ifakara, Tanzania. J Clin Microbiol. 1999;37:3113-7.

31. Gu B, Cao Y, Pan S, Zhuang L, Yu R, Peng Z, et al. Comparison of the prevalence and changing resistance to nalidixic acid and ciprofloxacin of Shigella between EuropeAmerica and Asia-Africa from 1998 to 2009. Int J Antimicrob Agents. 2012;40:9-17. http://dx.doi.org/10.1016/j.ijantimicag. 2012.02.005

32. Ruekit S, Wangchuk S, Dorji T, Tshering KP, Pootong $\mathbf{P}$, Nobthai $\mathbf{P}$, et al. Molecular characterization and PCRbased replicon typing of multidrug resistant Shigella sonnei isolates from an outbreak in Thimphu, Bhutan. BMC Res Notes. 2014;7:95. http://dx.doi.org/10.1186/1756-0500-7-95

33. Khan WA, Griffiths JK, Bennish ML. Gastrointestinal and extra-intestinal manifestations of childhood shigellosis in a region where all four species of Shigella are endemic. PLoS One. 2013;8:e64097. http://dx.doi.org/10.1371/journal.pone. 0064097

34. Markham KB, Backes C Jr, Samuels P. Bacteremia and intrauterine infection with Shigella sonnei in a pregnant woman with AIDS. Arch Gynecol Obstet. 2012;286:799-801. http://dx.doi.org/10.1007/s00404-012-2310-x

35. Koh X, Chiou C, Ajam N, Watanabe H, Ahmad N, Thong K. Characterization of Shigella sonnei in Malaysia, an increasingly prevalent etiologic agent of local shigellosis cases. BMC Infect Dis. 2012,12:122. http://dx.doi.org/10. 1186/1471-2334-12-122.

36. De Lappe N, Doran G, Connor JO, Mammina C, Cormican M. Use of pulsed-field gel electrophoresis for comparison of similar but distinguishable isolates of Shigella sonnei collected in Ireland and Italy. J Clin Microbiol. 2006;44: 3808-10. http://dx.doi.org/10.1128/JCM.01286-06 